Check for updates

Cite this: RSC Adv., 2019, 9, 40248

Received 9th October 2019

Accepted 26th November 2019

DOI: $10.1039 / c 9 r a 08227 a$

rsc.li/rsc-advances

\section{TADF and exciplex emission in a xanthone- carbazole derivative and tuning of its electroluminescence with applied voltage $\uparrow$}

\author{
Qamar T. Siddiqui, ${ }^{\text {ac }}$ Ankur A. Awasthi, ${ }^{\text {a }}$ Prabhjyot Bhui, ${ }^{\text {b }}$ Pradnya Parab, \\ Mohammad Muneer, (D) ${ }^{c}$ Sangita Bose (D)*b and Neeraj Agarwal (D)*a
}

\begin{abstract}
Materials showing white light emission have found applications in a variety of solid state devices especially in display technology. For white light emission, doping of red (R), green (G) and blue (B) emitters in a host matrix is commonly practised. However, finding RGB emitters of similar stability with homogenous doping is challenging. Furthermore, such devices suffer from color purity in the long run. Small organic light emitters, capable of colour tuning and having a broad emission spectrum are in high demand as they provide colour stability, reproducibility, a simple device geometry and high efficiency. Recently, it has been shown that the efficiency of OLEDs can be enhanced by employing thermally activated delayed fluorescence (TADF) materials. Here, we designed and synthesised a xanthone-carbazole based D-A-D material (Xan-Cbz) for TADF properties. Blue TADF emission, in neat thin films, at $470 \mathrm{~nm}$ was observed and further investigated by studying delayed fluorescence and lifetime measurements. In addition, a blend of Xan-Cbz with NPD shows exciplex emission at $525 \mathrm{~nm}$ in thin film. OLEDs based on Xan-Cbz were fabricated using several device configurations. OLEDs having the device configuration ITO/PEDOT:PSS/NPD/Xan-Cbz/Bphen/LiF-Al showed a luminance of $1.96 \times 10^{4} \mathrm{Cd} \mathrm{m}^{-2}$ (at a current density of $50 \mathrm{~mA} \mathrm{~cm}{ }^{-2}$ ) and $V_{O N}$ at $\sim 6 \mathrm{~V}$. Electroluminescence showed the features of both neat emission (470 nm) of Xan-Cbz and its exciplex (525 nm) with NPD. Further, colour tuning was observed as a function of applied voltage and the ratio of light intensity $\left(/_{525} / /_{470}\right)$ of neat and exciplex emission was found to decrease with increasing voltage. Greenish-blue emission (CIE coordinates: 0.202, 0.382) from Xan-Cbz OLEDs was obtained. Xan-Cbz showed its neat emission (at $470 \mathrm{~nm}$ ) in ITO/PEDOT:PSS/ CBP/Xan-Cbz/Bphen/LiF-Al and pure exciplex emission (at $525 \mathrm{~nm}$ ) in ITO/PEDOT:PSS/NPD:Xan-Cbz/ Bphen/LiF-Al device configurations. Thus in this article we showed blue TADF emission, exciplex emission and voltage dependent color tuning in OLEDs based on a small organic emitter.
\end{abstract}

\section{Introduction}

Organic light emitting devices (OLEDs) are popular for activeluminescent displays and lighting technologies, owing to their special features, such as easy processing, light weight, flexibility, commercial viability etc. ${ }^{\mathbf{1 - 1 2}}$ For white light, emission of primary colours (RGB) or a broad visible-light spectrum is vital. Several strategies have been reported by various groups to obtain white light (Fig. 1). Commonly, premeditated mixing of blue, green and red emitters or blue and orange emitters has

\footnotetext{
${ }^{a}$ School of Chemical Sciences, UM-DAE, Centre for Excellence in Basic Sciences, University of Mumbai, Santacruz (E), Mumbai, 400098, India. E-mail: na@cbs.ac.in ${ }^{b}$ School of Physical Sciences, UM-DAE, Centre for Excellence in Basic Sciences, University of Mumbai, Santacruz (E), Mumbai, 400098, India. E-mail: sangita@cbs. ac.in

${ }^{c}$ Department of Chemistry, Aligarh Muslim University, Aligarh, India

$\dagger$ Electronic supplementary information (ESI) available. See DOI: 10.1039/c9ra08227a
}

been applied in devices to obtain white light OLEDs. ${ }^{13-20}$ In such devices, emitters of divergent colours are doped in a suitable host matrix especially when phosphorescent materials are in action. Use of several emitters and hosts make the device architecture very complex thus causing tedious device fabrication. Tandem OLEDs have been considered for multi coloured emission by using polymer as well as small organic molecules. ${ }^{\mathbf{2 1 2 2}}$ Such OLEDs provide easy accessibility of broad/white light emission; however, the device geometry becomes complicated as an additional electrode is required. In few devices reported in the literature, processes like exciplex formation and Auger recombination has been shown leading to variable colour emission depending on the applied voltage. ${ }^{23,24}$

To simplify the device fabrication process, single organic light emitters which are capable of colour tuning and broad emission spectrum are essential. Single emitter with tunability in OLED is advantageous as it provides colour stability, reproducibility, and simple device geometry. To facilitate this, new molecules have to be designed which are capable of producing emission in wide 


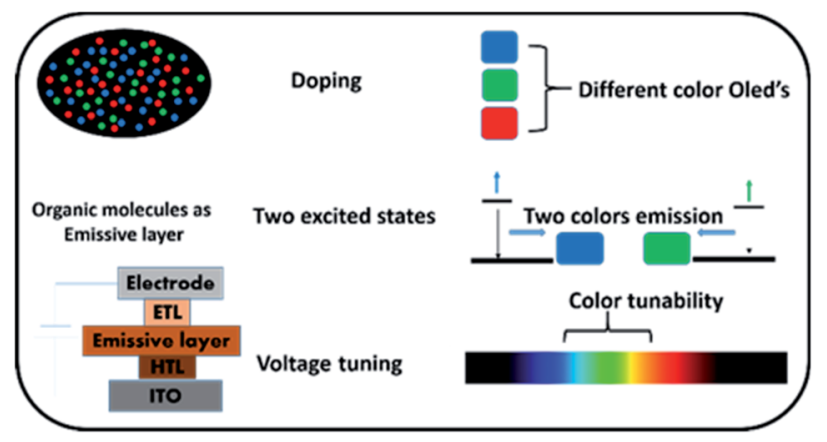

Fig. 1 Various strategies for multi-coloured emission from OLEDs.

visible region. More than one emission from single molecule can be achieved by having radiative decay from two different excited states or formation of excimer and exciplex emission in solid state. In order to improve the efficiency of OLEDs, involvement of singlet and triplet excited states is advantageous. This can be achieved by employing the phosphorescent emitters, with molecules having thermally activated delayed fluorescence (TADF) or triplet-triplet annihilation (TTA) features. ${ }^{25-32}$ We recently reported TADF in undoped acridone-carbazole derivatives and their exciplex emission in OLEDs. ${ }^{33}$ Generally, exciplex is studied in the blend of suitable donor and acceptor molecules to achieve new emission different than that of individual donor and acceptor materials. In devices, when hole transporting materials capable of forming exciplex with emitter is placed beside it, formation of exciplex at the interface of two layers can be expected. In the same device emission from neat emitter can also be obtained when recombination of excitons happens in the emissive layer. Exciton recombination depends on several factors e.g. alignment of energy levels of different materials used in the device, charge carrier mobilities, thickness of layers, applied voltage etc. Suitable device geometry can help in obtaining the emission from neat emitter and exciplex simultaneously. Here, in this manuscript, we report on the design and synthesis of xanthone-carbazole (Xan-Cbz) derivative which shows blue TADF $(\sim 470 \mathrm{~nm})$ in undoped film and an exciplex emission ( $\sim 525 \mathrm{~nm})$ when blended with NPD ( $N, N^{\prime}$-di(1-naphthyl)$N, N^{\prime}$-diphenyl-(1,1'-biphenyl)-4,4'-diamine). OLEDs of Xan-Cbz were fabricated in different device geometries to ascertain which of the two emissions ( $\sim 470$ or $\sim 525 \mathrm{~nm}$ ) is more dominant in the device. It was observed that for the device architecture where NPD was used as a hole transporting layer prior to the active layer, exciplex emission at $525 \mathrm{~nm}$ was obtained from the interface, accompanied by TADF emission from the active layer of Xan-Cbz. Interestingly, for such devices, the ratio of light from the neat emitter (Xan-Cbz) and its exciplex was seen to be tunable with the applied voltage. Thus, our results show that Xan-Cbz can be used as a green or a blue emitter depending on the bias voltage in the OLED device.

\section{Results and discussion}

Recently, we have reported palladium free amination of acridone with carbazole using 1,10-phenanthroline and copper iodide as catalysing agents. ${ }^{32}$ Xan-Cbz was synthesized in a similar way (see Scheme S1 of the ESI†). In this reaction, mixture of mono- and di amines were formed along with unreacted carbazole. In column chromatography, carbazole and Xan-Cbz moved together as they have identical retention factor (Rf). For further purification chemical method was used (see ESI $\dagger$ ) followed by column chromatography and recrystallization. Characterization of Xan-Cbz was carried out by several spectroscopic techniques e.g. ${ }^{1} \mathrm{H}-\mathrm{NMR},{ }^{13} \mathrm{C}-\mathrm{NMR}$, MALDI-TOF (see ESI $\dagger$ ).

Ground and excited state photophysical properties of XanCbz was studied by using absorption and emission spectroscopies in both solution and thin films. Absorption and emission spectra of Xan-Cbz, in dichloromethane and thin films, were recorded and are shown in Fig. 2. Photophysical data is summarized in Table 1 . In absorption spectra, a low intensity broad peak was observed at $370 \mathrm{~nm}$ for Xan-Cbz. Two high intensity peaks at $265-310 \mathrm{~nm}$ were also observed. The broad, low intensity peak is attributed to charge transfer band and further explored by solvatochromic studies. A slight shift is observed in absorption spectra in various polarity solvents. Contrary to absorption spectra, there is a significant red shift of $\sim 90 \mathrm{~nm}$ between low polarity (Toluene, $470 \mathrm{~nm}$ ) and high polarity (DMSO, $560 \mathrm{~nm}$ ) solvents in the emission spectra. It is well known that the emission spectra arising from locally exited (LE) $S_{1}-S_{0}$ does not show significant shift with increasing

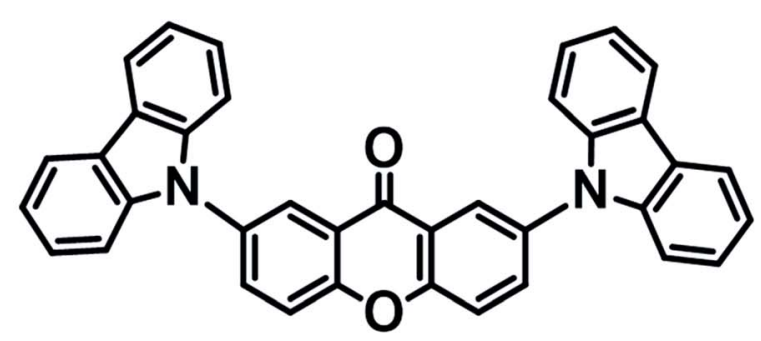

(a)

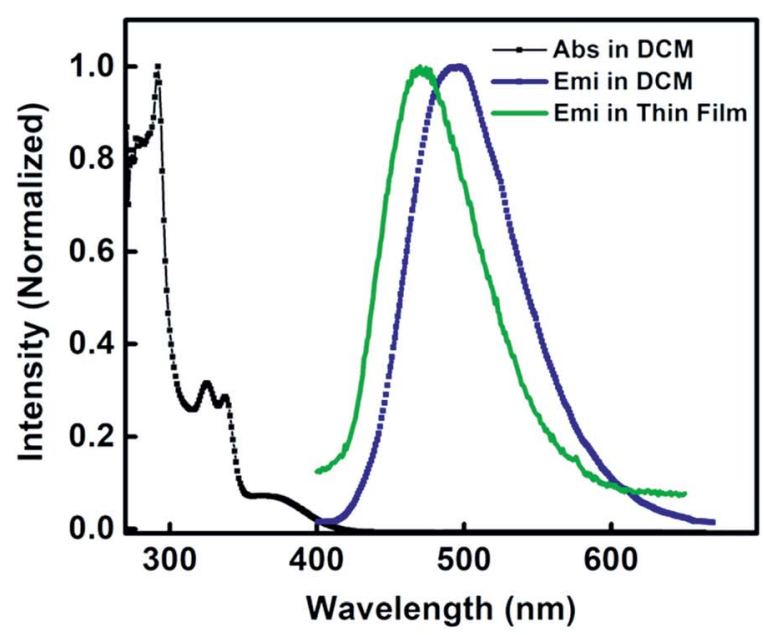

(b)

Fig. 2 (a) Molecular structure of Xan-Cbz and (b) absorption, emission spectra in DCM and emission spectrum in thin film. 
Table 1 Photophysical and electrochemical data of Xan-Cbz

\begin{tabular}{|c|c|c|c|c|c|c|c|c|c|c|c|}
\hline$\lambda_{\mathrm{abs}}{ }^{a} \mathrm{~nm}(\log \varepsilon)$ & $\begin{array}{l}\lambda_{\mathrm{abs}}{ }^{b} \\
(\mathrm{~nm})\end{array}$ & $\begin{array}{l}\lambda_{\mathrm{em}}{ }^{a} \\
(\mathrm{~nm})\end{array}$ & $\begin{array}{l}\lambda_{\mathrm{em}}{ }^{b} \\
(\mathrm{~nm})\end{array}$ & $\begin{array}{l}E_{\text {номо }}{ }^{c} \\
(\mathrm{eV})\end{array}$ & $\begin{array}{l}E_{\text {LUMO }}{ }^{d} \\
(\mathrm{eV})\end{array}$ & $\phi$ & $\begin{array}{l}\tau_{\mathrm{PF}} \\
(\mu \mathrm{s})\end{array}$ & $\begin{array}{l}\tau_{\mathrm{TADF}} \\
(\mu \mathrm{s})\end{array}$ & $\begin{array}{l}E_{\mathrm{S} 1} \\
(\mathrm{eV})\end{array}$ & $\begin{array}{l}E_{\mathrm{T} 1} \\
(\mathrm{eV})\end{array}$ & $\begin{array}{l}\Delta E_{\mathrm{ST}} \\
(\mathrm{eV})\end{array}$ \\
\hline $\begin{array}{l}292 \text { (4.13), } 325 \text { (4.14), } \\
338 \text { (4.09), } 370 \text { (3.48) }\end{array}$ & 387 & 493 & 470 & 6.0 & 3.0 & 22 & $\begin{array}{l}0.011 \text { (in air), } \\
0.016 \text { (in } \mathrm{N} 2 \text { ) }\end{array}$ & 3.8 & 2.42 & 2.10 & 0.32 \\
\hline
\end{tabular}

polarity whereas the emission spectra arising from charge transfer (CT) states are broad and shows dependence on polarity of the solvent medium. ${ }^{34-38}$ Solvent dependent studies of Xan-Cbz suggest that the emission maxima is arising from ${ }^{1} \mathrm{CT}$ transition. The emission maxima for Xan-Cbz in dichloromethane was found to be at $493 \mathrm{~nm}$ and a hypsochromic shift of $\sim 20 \mathrm{~nm}$ was observed in thin films $(470 \mathrm{~nm})$.

For the TADF prospects, the difference in the singlet and triplet energy levels $\left(\Delta E_{\mathrm{ST}}\right)$ is required to be estimated. It is known that triplet states quenches at room temperature, therefore, phosphorescence emission was recorded at $77 \mathrm{~K}$ in 2methyltetrahydrofuran (2-MeTHF). To populate the triplet state ethyl iodide $(10 \% \mathrm{v} / \mathrm{v})$ was added to the solvent as it sensitizes the triplet state by heavy atom effect. Emission spectra of Xan$\mathrm{Cbz}$ at low temperature in presence of ethyl iodide is shown in ESI. $\uparrow$ Xan-Cbz showed two peaks at 510 and $590 \mathrm{~nm}$. Peak at $\sim 510 \mathrm{~nm}$ is assigned to fluorescence emission and the new peak at $\sim 590 \mathrm{~nm}$ is accounted for phosphorescence. Singlet and triplet energy levels were estimated from the peak maxima of fluorescence and phosphorescence peaks respectively. Singlet and triplet energy levels of Xan-Cbz are summarized in Table 1. From $E_{\mathrm{S} 1}$ and $E_{\mathrm{T} 1}, \Delta E_{\mathrm{ST}}$ was found to be $0.32 \mathrm{eV}$. Energy gap between singlet and triplet energy levels is similar to those of TADF materials recently used in OLEDs. ${ }^{39-41}$ Thus, we believe that these materials can find applications in OLEDs.

In order to use Xan-Cbz in solid state devices it is important to find out the highest occupied molecular orbital ( $\left.E_{\text {номо }}\right)$ and lowest unoccupied molecular orbital ( $E_{\text {LUMO }}$ ) energy levels. The $E_{\mathrm{HOMO}}$ and $E_{\mathrm{LUMO}}$ were estimated using the cyclic voltammetry measurements and optical band gap. For organics, first oxidation potential can be considered as its HOMO energy level. The first oxidation potential $\left(E_{\mathrm{ox}}\right)$ of Xan-Cbz with respect to oxidation potential of ferrocene $\left(\mathrm{Fc} / \mathrm{Fc}^{+}\right.$, as internal standard) was found out. $E_{\mathrm{ox}}$ was observed at $0.9 \mathrm{~V}$ higher than that of $\mathrm{Fc} / \mathrm{Fc}^{+}$ (see ESI $\dagger$ ). Using $E_{\text {HOMO }}=E_{\text {ox }}$ w.r.t Fc/Fc ${ }^{+}+5.1 \mathrm{eV}^{42-44} \mathrm{HOMO}$ energy level was estimated to be $-6.0 \mathrm{eV}$. The optical gap, as measured from the intersection of absorption and emission bands of Xan-Cbz is $\sim 3.0 \mathrm{eV}$. Using $E_{\mathrm{HOMO}}$ and optical gap, $E_{\text {LUMO }}$ were estimated at $\sim-3.0 \mathrm{eV}$.

To establish the TADF property in Xan-Cbz fluorescence decay was studied in several experimental conditions. Transient measurement at room temperature showed single exponential decay arising from prompt fluorescence (Fig. 3). Fluorescence life times observed for Xan-Cbz is $\sim 11 \mathrm{~ns}$. Difference between singlet and triplet energy levels of Xan-Cbz is small, therefore, triplet state may also contribute to emission lifetime as it happens in TADF materials. To get an idea about the contribution of triplet state, life time studies were also carried out under nitrogen purged solvent as it eliminates the triplet quenching due to the presence of oxygen and therefore enhances the utilization of triplet states in emission. Life times for Xan-Cbz in nitrogen purged solvent increased by $\sim 50 \%$ and observed to be $\sim 16$ ns (Fig. 3). These observations further suggest the close proximity of singlet and triplet energy levels. ${ }^{40,45}$

The life time of triplet state is generally in microsecondmillisecond ( $\mu \mathrm{s}-\mathrm{ms})$ range. Long life time of triplet states may results in triplet-triplet annihilation which is not as efficient as

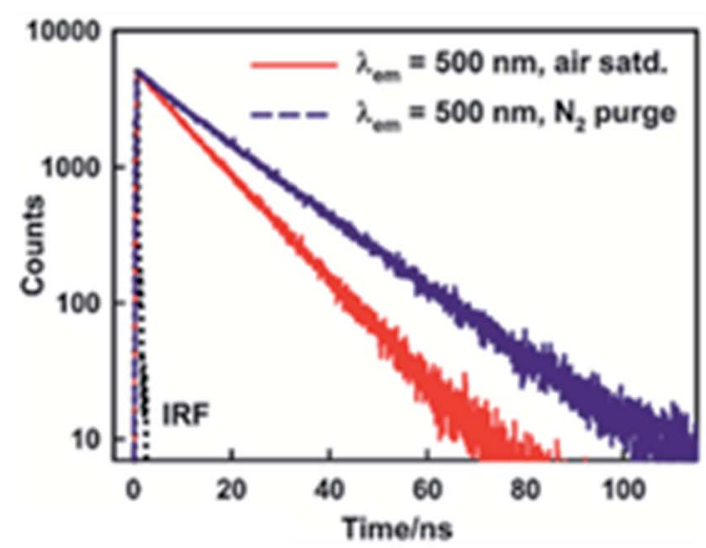

(a)

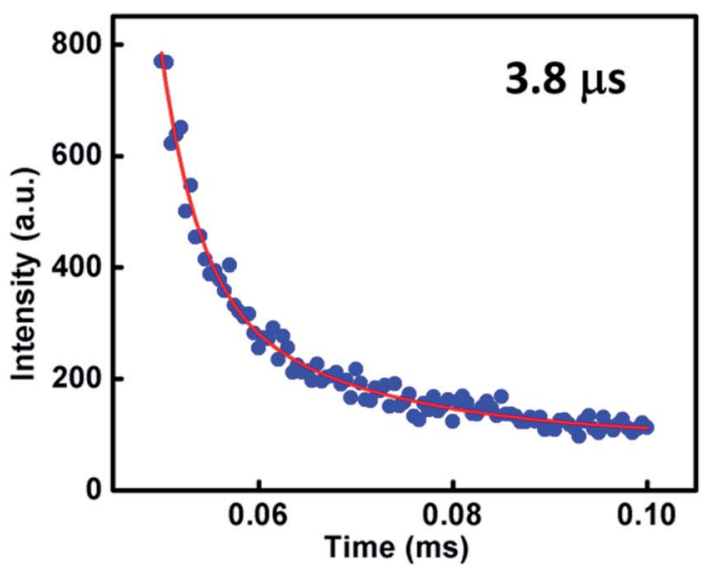

(b)

Fig. 3 Fluorescence decay profiles of Xan-Cbz in (a) air saturated and nitrogen purged THF (b) in thin film. 
TADF. ${ }^{4-51}$ Hence, it is desirous to have short triplet life time to have better TADF which should also results in short TADF life time. We determined the TADF life time of Xan-Cbz in undoped thin films. The fluorescence decay profile (at $470 \mathrm{~nm}$ ) were recorded after a delay of $50 \mu \mathrm{s}$. TADF life time for Xan-Cbz was estimated to be $3.8 \mu \mathrm{s}$. As mentioned earlier, the prompt fluorescence life time is much less than 100 ns (Table 1), hence, spectra obtained after a delay of $50 \mu$ s rules out the possibility of any component due to prompt fluorescence. Thus, emission decay obtained after a delay is attributed to TADF.

In blends of electron donor and acceptor, excited state formation by electron transfer from donor to acceptor can be envisaged. Such excited states are, generally, composed of excited state of donor and ground state of acceptor molecule and are called as exciplex. Exciplex shows different emission from both of its components i.e. it does not match with the individual emission of donor and/or acceptor. Also, exciplex emission is found to be broader and structure less. It is important to have suitable HOMO and LUMO energy levels of donor and acceptor as they play an important role in charge transfer and thus in exciplex formation. It has been suggested that HOMO and LUMO of donor should be more than that of acceptor. For efficient charge transfer the energy difference between the LUMOs of donor and acceptor should be at least $0.5 \mathrm{eV}$ as lesser difference will cause energy transfer. We studied the exciplex formation in blend of Xan-Cbz with NPD and CBP (4,4'-bis-( $N$-carbazolyl)-1,1'-biphenyl). In the blend with NPD, NPD acts as electron donor while Xan-Cbz as acceptor. Emission of blend of NPD with Xan-Cbz is shown in Fig. 4. A new emission peak is observed with maxima at $525 \mathrm{~nm}$ which does not belong to either NPD or Xan-Cbz. This new peak is assigned to exciplex emission. Fluorescence lifetime of this new emission is also recorded (see Fig. $\mathrm{S} 7 \dagger$ ). Long life time is an indication of involvement of TADF in exciplex formation. No exciplex formation was observed for the blend of Xan-Cbz with CBP as HOMO and LUMO energy levels of CBP are not compatible for the exciplex formation with Xan-Cbz.

To study the prospects of Xan-Cbz in OLEDs, devices in different geometries were fabricated and studied. One of the



Fig. 4 Emission spectra of Xan-Cbz, NPD and its blend $(1: 1)$ in thin films. device geometry which was studied was ITO/PEDOT:PSS/NPD/ Xan-Cbz/Bphen/LiF-Al. In this device, poly(2,3-dihydrothieno1,4-dioxin)-poly(styrenesulfonate) (PEDOT:PSS) was used as a HTL. Since, $E_{\text {Hомо }}$ of NPD is very close to $E_{\text {Hомо }}$ of Xan-Cbz, a thin layer of NPD $(\sim 40 \mathrm{~nm})$ was used in between the PEDOT:PSS and Xan-Cbz which was expected to further facilitate the hole transport. Bathophenanthroline (Bphen) was used as a hole blocking layer (HBL). In these devices, while PEDOT:PSS was spin coated with the usual protocol to give $\sim 50 \mathrm{~nm}$ films, the rest of the layers were thermally evaporated under vacuum (with a base vacuum of $1 \times 10^{-6} \mathrm{mbar}$ ). The active area of the devices were in the range of $0.05-0.08 \mathrm{~cm}^{2}$. Turn on voltage, $V_{\text {ON }}$ for this geometry was found to be $\sim 6 \mathrm{~V}$. Bright light was obtained showing a luminance as high as 1.96 $\times 10^{4} \mathrm{Cd} \mathrm{m}^{-2}$ (@ a current density of $50 \mathrm{~mA} \mathrm{~cm}^{-2}$ ) as can be seen from the device characteristics shown in Fig. 5(a). Furthermore, an interesting phenomenon was observed in these devices. At biases of 6-12 V, the colour of the emission from the devices visually appeared predominantly green. However, at higher biases $(12-20 \mathrm{~V})$, the devices appeared bluish green. The electroluminescence spectra at different voltages is shown in Fig. 5(b). Here, the EL spectra are shown for different bias voltages which are normalized with the light intensity recorded at $9 \mathrm{~V}$ at $525 \mathrm{~nm}$. Two important points can be identified from the EL spectra. (i) At all voltages two peaks are seen at $\sim 465 \mathrm{~nm}$ which matches with the emission of Xan$\mathrm{Cbz}$ in thin films (see Fig. 4) and at $\sim 525 \mathrm{~nm}$ which matches with the PL emission (exciplex) of blend of Xan-Cbz and NPD (see Fig. 4). (ii) The ratio of the light intensity at 525 and $465 \mathrm{~nm}$, is seen to decrease with increasing bias voltage which implies a voltage tunability of the light emission in these devices. In these devices, the exciplex is formed only at the narrow interface of NPD and Xan-Cbz. At lower voltages, exciplex emission is dominant, which can be explained by assuming that lesser number of carriers transported to the active layer (Xan-Cbz) and hence the exciplex formation at the interface is facilitated. As expected, with increase of applied voltage, more carriers will reach the active layer of Xan-Cbz. Thus, exciton recombination occurs in the active layer of Xan-Cbz as well as at the interface of Xan-Cbz and NPD at higher voltages. Emission from neat Xan$\mathrm{Cbz}$ and its exciplex with NPD (at interface) leads to broader spectral range of light from OLED. Colour tuning of device with applied voltage is clearly visible from the colour gamut showing the CIE coordinates of the emission (Fig. 5(c)).

As discussed earlier from the photophysical studies of Xan$\mathrm{Cbz}$ and its blend with NPD in thin film, the peak at $\sim 465 \mathrm{~nm}$ was attributed to the TADF emission of Xan-Cbz and the peak at $525 \mathrm{~nm}$ was assigned for exciplex emission. To further verify the exciplex formation under electrical excitation, devices of two different geometries were fabricated. In the first geometry, ITO/ PEDOT:PSS/Xan-Cbz + NPD (1:1)/Bphen/LiF-Al, having blend of Xan-Cbz and NPD as active emitting layer. The device characteristics shown in Fig. 6(a) shows reasonable light emission $(L$ $=2890 \mathrm{Cd} \mathrm{m}^{-2}$ @ $50 \mathrm{~mA} \mathrm{~cm}{ }^{-2}$ ) with a $V_{\mathrm{ON}}=7.7 \mathrm{~V}$. The EL spectra of this device (Fig. 6(b)) indeed showed a dominant emission at $525 \mathrm{~nm}$ consistent with that of the PL observed in thin film. Peak at $525 \mathrm{~nm}$ in EL is thus attributed to exciplex 


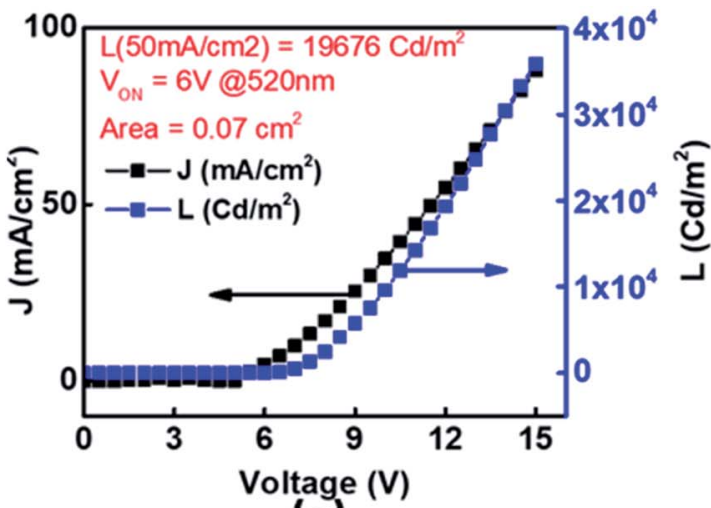

(a)

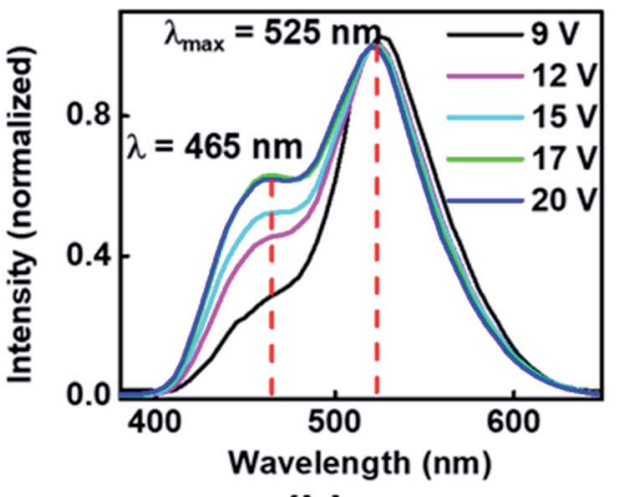

(b)

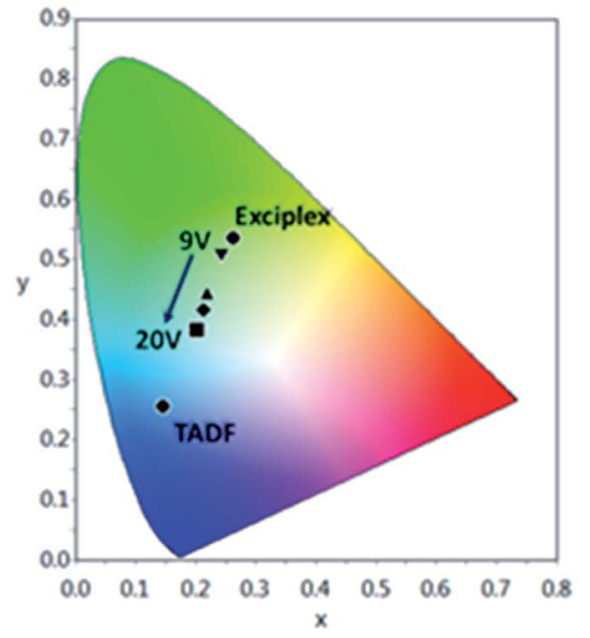

(c)

Fig. 5 (a) Device, ITO/PEDOT:PSS/NPD/Xan-Cbz/Bphen/LiF-Al, characteristics ( $J$ and $L$ vs. bias voltage) of Xan-Cbz; (b) normalized electroluminescence of the device at different voltages and (c) color gamut showing the emission color at different voltages.

formation under electrical excitation. This device showed very little (negligible) effect of varying voltage on EL spectra. In other device CBP was used in place of NPD and the device structure is: ITO/PEDOT:PSS/CBP/Xan-Cbz/Bphen/LiF-Al. Here, CBP was used as an additional HTL. It is worth to mention that blend of Xan-Cbz and CBP $(1: 1)$ does not show exciplex formation as discussed earlier. Fig. 6(c) shows the device characteristics.
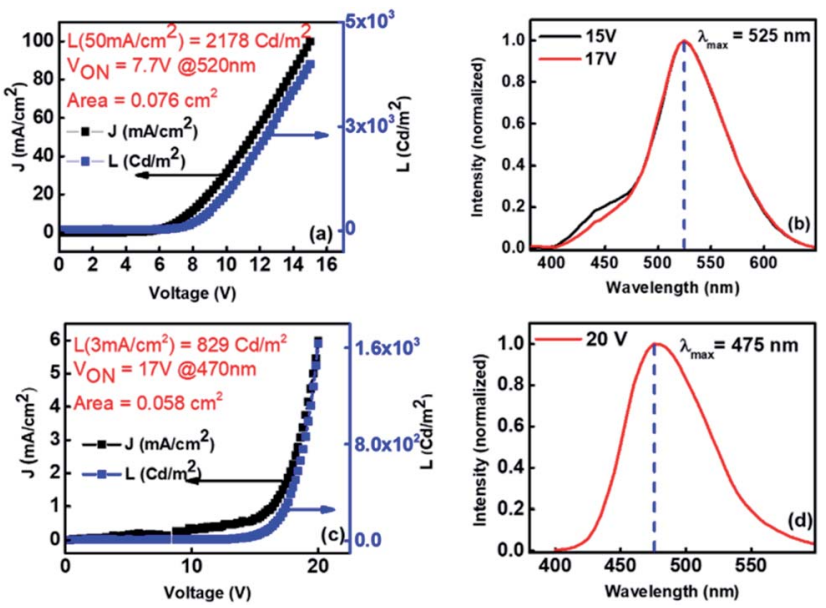

Fig. 6 (a) Device, ITO/PEDOT:PSS/NPD:Xan-Cbz/Bphen/LiF-Al, characteristics ( $J$ and $L$ vs. bias voltage) of exciplex system formed using a blend of Xan-Cbz and NPD (1: 1 by weight); (b) EL of exciplex forming device at different voltages; (c) $J-L$ characteristics of device, ITO/PEDOT:PSS/CBP/Xan-Cbz/Bphen/LiF-Al; and (d) its EL.

Maxima in electroluminescence was obtained at $475 \mathrm{~nm}$ which corresponds to emission of Xan-Cbz (Fig. 6(d)). However, these devices seemed to be less efficient than the previous two. Both the current density and light intensity were low from these devices and the $V_{\mathrm{ON}}$ was quite high (see Fig. 6(c)).

\section{Experimental}

\section{Materials and methods}

Chemicals were purchased from Sigma-Aldrich and used as received. Organic solvents were dried using reported standard procedures. ${ }^{1} \mathrm{H}$ and ${ }^{13} \mathrm{C}$ NMR spectra were recorded using a Bruker $300 \mathrm{MHz}$ spectrometer. Tetramethylsilane (TMS) in $\mathrm{CDCl}_{3}$ was used as an internal reference residual proton; $\delta=$ $7.26 \mathrm{ppm}$. Mass spectra were recorded on Bruker MALDI-TOF instrument. $\mathrm{CH}$ Instruments 620D electrochemical analyser was used to perform cyclic voltammetry. Anhydrous acetonitrile with tetrabutylammonium hexafluorophosphate solution $(0.1$ $\mathrm{M})$ as supporting electrolyte at room temperature was taken for measurement. The oxidation potential of $\mathrm{Fc} / \mathrm{Fc}^{+}$was used as internal reference. The absorption spectra were recorded using Shimadzu 1800, and emission spectra were recorded on Agilent, Fluorolog-3 and Fluoromax-4 spectrofluorometer. A diode laserbased time-correlated single-photon counting (TCSPC) spectrometer (IBH, U.K.) was used to obtain the time-resolved fluorescence measurements and has been described in detail elsewhere. TADF lifetime decay was observed after a delay of 50 $\mu$ s on Horiba Fluorolog-3 Spectrofluorometer. Thin films for emission studies were prepared by spin coating using Holmarc HO-TH-05 spin coater at $2000 \mathrm{rpm}$ for $40 \mathrm{~s}$. For OLED device fabrication, PEDOT:PSS was spin coated at $8000 \mathrm{rpm}$ for $40 \mathrm{~s}$. Vacuum deposition was carried out at a base vacuum of $2 \times$ $10^{-6}$ mbar. ITO-coated substrate (15-25 $\Omega \mathrm{sq}^{-1}$, Sigma-Aldrich) was cut into $22 \times 12 \mathrm{~mm}^{2}$. Desired pattern were etched on the ITO substrate using $\mathrm{Zn}$ powder and $10 \% \mathrm{HCl}$. Substrate was first cleaned with soap solution and distilled water. Then it was 
sonicated for $10 \mathrm{~min}$ each in distilled water and isopropanol. In the last step substrate was cleaned with trichloroethylene vapours. After cleaning, the ITO coated substrate is given UV treatment for $1 \mathrm{~h}$ after which the substrates were ready for device fabrication. CBP, NPD, Xan-Cbz, Bphen, $\mathrm{LiF}$ and $\mathrm{Al}$ where ever required were thermally evaporated in vacuum (base vacuum $\sim 10^{-6} \mathrm{mbar}$ ). For exciplex devices a homogenous solution of NPD + Xan-Cbz (1 mg each) is made in chloroform $(0.5 \mathrm{~mL})$ and spin coated at $3000 \mathrm{rpm}$ for $40 \mathrm{~s} . J-V$ measurements of the OLED devices were carried out using a 2400 Keithley sourcemeter at the emitting wavelength. Using lock-in detection the luminance-voltage $(L-V)$ spectra were also simultaneously recorded. The lock-in signal was converted to light units using the conversion factors, which were estimated using a calibrated light source. Electroluminescence of the devices were measured on a setup consisting of a Bausch and Lomb 350-750 nm monochromator and a Hamamatsu R212 photomultiplier tube as the detector.

\section{Conclusions}

Xanthone-carbazole based organic D-A-D molecule Xan-Cbz was synthesized by substituting carbazole on 2, 7 position of xanthone core. Photophysical studies were performed to establish the TADF in Xan-Cbz. Thin film studies of blends of Xan-Cbz with NPD showed the formation of exciplex. OLED of Xan-Cbz were fabricated using the device architecture as ITO/PEDOT:PSS/NPD/Xan$\mathrm{Cbz} / \mathrm{Bphen} / \mathrm{LiF}-\mathrm{Al}$. The turn on voltage $\left(V_{\mathrm{ON}}\right)$ was $\sim 6 \mathrm{~V}$ and luminous intensity was found to be $1.96 \times 10^{4} \mathrm{Cd} \mathrm{m^{-2 }}$ at a current density of $50 \mathrm{~mA} \mathrm{~cm}{ }^{-2}$. Electroluminescence peaks at 465 and $525 \mathrm{~nm}$ were observed which were attributed to emission from neat Xan-Cbz and its exciplex (with NPD) respectively. With increasing voltage the ratio of two peaks changed and colour of device changed from green to greenish blue. Pure TADF and pure exciplex devices were also fabricated and studied.

\section{Conflicts of interest}

There are no conflicts to declare.

\section{Acknowledgements}

We thank Swati Dixit for assistance in cyclic voltammetric studies. We thank Tata Institute of Fundamental Research, Mumbai for NMR, MALDI-TOF and photophysical studies on Fluorolog-3. We also thank Radiation and Photochemistry Division, Bhabha Atomic Research Centre for TCSPC. NA and SB thank Department of Science and Technology for partial financial support (EMR/2017/000805).

\section{References}

1 C. J. Chiang, A. Kimyonok, M. K. Etherington, G. C. Griffiths, V. Jankus, F. Turksoy and A. P. Monkman, Adv. Funct. Mater., 2013, 23, 739-746.

2 S. R. Forrest, Nature, 2004, 428, 911-918.
3 S. R. Forrest, M. A. Baldo, D. F. O'Brien, Y. You, A. Shoustikov, S. Sibley and M. E. Thompson, Nature, 1998, 395, 151-154.

4 N. Agarwal, P. K. Nayak, F. Ali, M. P. Patankar, K. L. Narasimhan and N. Periasamy, Synth. Met., 2011, 161, 466-473.

5 P. Bhui, Q. T. Siddiqui, M. Muneer, N. Agarwal and S. Bose, J. Chem. Sci., 2018, 130, 167.

6 M.-K. Fung, Y.-Q. Li and L.-S. Liao, Adv. Mater., 2016, 28, 10381-10408.

7 S. Y. Lee, T. Yasuda, Y. S. Yang, Q. Zhang and C. Adachi, Angew. Chem., Int. Ed., 2014, 53, 6402-6406.

8 K. Nasu, T. Nakagawa, H. Nomura, C.-J. Lin, C.-H. Cheng, M.-R. Tseng, T. Yasuda and C. Adachi, Chem. Commun., 2013, 49, 10385-10387.

9 P. K. Nayak, N. Agarwal, F. Ali, M. Patankar, N. Periasamy and K. L. Narasimhan, J. Chem. Sci., 2010, 122, 847.

10 R.-P. Xu, Y.-Q. Li and J.-X. Tanga, J. Mater. Chem. C, 2016, 4, 9116-9142.

11 Q. Wu, M. Wang, X. Cao, D. Zhang, N. Sun, S. Wan and Y. Tao, J. Mater. Chem. C, 2018, 6, 8784-8792.

12 C. M. Marian, J. Phys. Chem. C, 2016, 120, 3715-3721.

13 T. Higuchi, H. Nakanotani and C. Adachi, Adv. Mater., 2015, 27, 2019-2023.

14 J. Kido, M. Kimura and K. Nagai, Science, 1994, 267, 1332.

15 G. W. Kim, H. W. Bae, R. Lampande, I. J. Ko, J. H. Park, C. Y. Lee and J. H. Kwon, Sci. Rep., 2018, 8, 5161.

16 W. Liu, C.-J. Zheng, K. Wang, M. Zhang, D.-Y. Chen, S.-L. Tao, F. Li, Y.-P. Dong, C.-S. Lee, X.-M. Ou and X.-H. Zhang, ACS Appl. Mater. Interfaces, 2016, 8, 3298432991.

17 S. Reineke, F. Lindner, G. Schwartz, N. Seidler, K. Walzer, B. Lüssem and K. Leo, Nature, 2009, 459, 234.

18 Y. Sun and S. R. Forrest, Appl. Phys. Lett., 2007, 91, 263503.

19 S. Wu, S. Li, Q. Sun, C. Huang and M.-K. Fung, Sci. Rep., 2016, 6, 25821.

20 P. Xiao, T. Dong, J. Xie, D. Luo, J. Yuan and B. Liu, Appl. Sci., 2018, 8, 299-331.

21 W.-Y. Hung, G.-C. Fang, S.-W. Lin, S.-H. Cheng, K. T. Wong, T.-Y. Kuo and P.-T. Chou, Sci. Rep., 2014, 4, 5161.

22 B. Zhao, T. Zhang, B. Chu, W. Li, Z. Su, Y. Luo, R. Li, X. Yan, F. Jin, Y. Gao and H. Wu, Org. Electron., 2015, 17, 15-21.

23 H. A. A. Attar and A. P. Monkman, Adv. Mater., 2016, 28, 8014-8020.

24 S.-J. He, D.-K. Wang, N. Jiang, J. S. Tse and Z.-H. Lu, Adv. Mater., 2016, 28, 649-654.

25 H. Kaji, H. Suzuki, T. Fukushima, K. Shizu, K. Suzuki, S. Kubo, T. Komino, H. Oiwa, F. Suzuki, A. Wakamiya, Y. Murata and C. Adachi, Nat. Commun., 2015, 6, 8476.

26 S. M. King, M. Cass, M. Pintani, C. Coward, F. B. Dias, A. P. Monkman and M. Roberts, J. Appl. Phys., 2011, 109, 074502.

27 Y.-L. Tung, S.-W. Lee, Y. Chi, L.-S. Chen, C.-F. Shu, F.-I. Wu, A. J. Carty, P.-T. Chou, S.-M. Peng and G.-H. Lee, Adv. Mater., 2005, 17, 1059-1064.

28 H. Uoyama, K. Goushi, K. Shizu, H. Nomura and C. Adachi, Nature, 2012, 492, 234-238. 
29 M. Y. Wong and E. Zysman-Colman, Adv. Mater., 2017, 29, 1605444.

30 J. Zhou, P. Chen, X. Wang, Y. Wang, Y. Wang, F. Li, M. Yang, Y. Huang, J. Yu and Z. Lu, Chem. Commun., 2014, 50, 75867589.

31 M. Flämmich, J. Frischeisen, D. S. Setz, D. Michaelis, B. C. Krummacher, T. D. Schmidt, W. Brütting and N. Danz, Org. Electron., 2011, 12, 1663-1668.

32 Y. Li, G. Xie, S. Gong, K. Wu and C. Yang, Chem. Sci., 2016, 7, 5441-5447.

33 Q. T. Siddiqui, A. A. Awasthi, P. Bhui, M. Muneer, K. R. S. Chandrakumar, S. Bose and N. Agarwal, J. Phys. Chem. C, 2019, 123, 1003-1014.

34 I. R. Gould, R. H. Young, L. J. Mueller and S. Farid, J. Am. Chem. Soc., 1994, 116, 8176-8187.

35 V. Martínez-Martínez, J. Lim, J. Bañuelos, I. López-Arbeloa and O. Š. Miljanić, Phys. Chem. Chem. Phys., 2013, 15, 18023-18029.

36 R. Nandy and S. Sankararaman, Beilstein J. Org. Chem., 2010, 6, 992-1001.

37 H. Noda, H. Nakanotani and C. Adachi, Sci. Adv., 2018, 4, eaao6910.

38 H. Song, K. Wang, Z. Kuang, Y. S. Zhao, Q. Guo and A. Xia, Phys. Chem. Chem. Phys., 2019, 21, 3894-3902.
39 K. Shizu, H. Noda, H. Tanaka, M. Taneda, M. Uejima, T. Sato, K. Tanaka, H. Kaji and C. Adachi, J. Phys. Chem. C, 2015, 119, 26283-26289.

40 Q. Zhang, B. Li, S. Huang, H. Nomura, H. Tanaka and C. Adachi, Nat. Photonics, 2014, 8, 326-332.

41 Q. Zhang, J. Li, K. Shizu, S. Huang, S. Hirata, H. Miyazaki and C. Adachi, J. Am. Chem. Soc., 2012, 134, 14706-14709.

42 G. Gritzner and J. Kuta, J. Pure Appl. Chem. Res., 1984, 56, 461-466.

43 B. K. Sharma, A. M. Shaikh, N. Agarwal and R. M. Kamble, RSC Adv., 2016, 6, 17129-17137.

44 S. Trasatti, Pure Appl. Chem., 1986, 58, 955-966.

45 H. Yersin, A. F. Rausch, R. Czerwieniec, T. Hofbeck and T. Fischer, Coord. Chem. Rev., 2011, 255, 2622-2652.

46 X. Cai, X. Li, G. Xie, Z. He, K. Gao, K. Liu, D. Chen, Y. Cao and S.-J. Su, Chem. Sci., 2016, 7, 4264-4275.

47 F. B. Dias, T. J. Penfold and A. P. Monkman, Methods Appl. Fluoresc., 2017, 5, 012001.

48 H. Fukagawa, T. Shimizu, N. Ohbe, S. Tokito, K. Tokumaru and H. Fujikake, Org. Electron., 2012, 13, 1197-1203.

49 D. Y. Kondakov, T. D. Pawlik, T. K. Hatwar and J. P. Spindler, J. Appl. Phys., 2009, 106, 124510.

50 Y. Luo and H. Aziz, Adv. Funct. Mater., 2010, 20, 1285-1293.

51 K. Masui, H. Nakanotani and C. Adachi, Org. Electron., 2013, 14, 2721-2726. 